\title{
Publisher Correction: Transition from fireball to Poynting-flux-dominated outflow in the three-episode GRB 160625B
}

B.-B. Zhang, B. Zhang, A. J. Castro-Tirado, Z. G. Dai, P.-H. T. Tam, X.-Y. Wang, Y.-D. Hu, S. Karpov, A. Pozanenko, F.-W. Zhang, E. Mazaeva, P. Minaev, A. Volnova, S. Oates, H. Gao, X.-F. Wu, L. Shao (D, Q.-W. Tang, G. Beskin, A. Biryukov (D), S. Bondar, E. Ivanov, E. Katkova, N. Orekhova, A. Perkov, V. Sasyuk, L. Mankiewicz, A. F. Żarnecki, A. Cwiek, R. Opiela, A. Zadrożny, R. Aptekar, D. Frederiks, D. Svinkin, A. Kusakin, R. Inasaridze, O. Burhonov, V. Rumyantsev (D), E. Klunko, A. Moskvitin, T. Fatkhullin, V. V. Sokolov, A. F. Valeev, S. Jeong, I. H. Park, M. D. Caballero-García, R. Cunniffe, J. C. Tello, P. Ferrero, S. B. Pandey, M. Jelínek, F. K. Peng, R. Sánchez-Ramírez and A. Castellón

Correction to: Nature Astronomy https://doi.org/10.1038/s41550-017-0309-8, published online 20 November 2017.

In the version of this Letter originally published, the letter í was mistakenly omitted from the surname of the author R. Sánchez-Ramírez. This has now been corrected. 